\title{
Factors affecting the morbidity and mortality of diverting stoma closure: retrospective cohort analysis of twelve-year period
}

\author{
Bojan Krebs, Arpad Ivanecz, Stojan Potrc, Matjaz Horvat \\ Department for Abdominal Surgery, University Clinical Centre Maribor, Slovenia
}

Radiol Oncol 2019; 53(3): 331-336.

Received 21 March 2019

Accepted 15 July 2019

Correspondence to: Bojan Krebs, M.D., Ph.D., Department for Abdominal Surgery, UCC Maribor, Ljubljanska ulica 5, SI-2000 Maribor, Slovenia. Phone: +386 40 425427; Fax: +386 2 3211257; E-mail: bojan.krebs@guest.arnes.si

Disclosure: No potential conflicts of interest were disclosed.

Background. Diverting stoma is often performed in rectal cancer surgery for reducing the consequences of possible anastomotic failure. Closing of stoma follows in most cases after a few months. The aim of our study was to evaluate morbidity and mortality after diverting stoma closure and to identify risk factors for complications of this procedure.

Patients and methods. At our department, we have performed a retrospective cohort analysis of data for 260 patients with diverting stoma closure from 2003 to 2015. Age, stoma type, patient's preoperative ASA score, surgical technique and time to stoma closure were investigated as factors which could influence the complication rate.

Results. 218 patients were eligible for investigation. Postoperative complications developed in 54 patients (24.8\%). Most common complications were postoperative ileus (10\%) and wound infection (5\%). Four patients died (1.8\%). There was no effect on complication rate regarding type of stoma, closing technique, patient's ASA status and patient age. The only factor influencing the complication rate was the time to stoma closure. We found that patients which had the stoma closed prior to 8 months after primary surgery had lower overall complication rate $(p<0.05)$. Conclusions. To reduce overall complication rate, our data suggest a shorter period than 8 months after primary surgery before closure of diverting stoma. As diverting stoma closure is not a simple operation, all strategies should be taken to reduce significant morbidity and mortality rate.

Key words: low anterior resection; surgical stoma closure; risk factors; morbidity; mortality

\section{Background}

There is no doubt that anastomotic leakage is one of the most important surgical complications of rectal surgery. ${ }^{1}$ Due to high morbidity and mortality, it is a major issue and is certainly affecting long-term survival. Clinically manifested anastomotic leaks are seen after $3-30 \%$ of low anterior resection for carcinoma and may even be associated with a higher local recurrence rate. ${ }^{2}$ The mortality rate associated with symptomatic anastomotic leaks varies between 6 and 22\%. ${ }^{3}$ Risk factors for anastomotic leakage are numerous and not wellexplored: neoadjuvant treatment, patient age, comorbidity, operation length, male sex, anastomotic height, peripheral arterial disease with different stages of reduced vascular supply after radiotherapy and systemic chemotherapy, and many others.

To avoid the severe consequences of anastomotic problems, it is crucial to take all available actions to prevent a symptomatic anastomotic leakage. One of the well-established procedures to prevent such a complication is diverting enterostomy. Nowadays, this is a common surgical choice to secure an anastomosis after low anterior resection for cancer, especially after neoadjuvant treatment. This procedure was also proposed by Working Group for Colon/Rectum Carcinoma (WGCRC) in 2002, who suggested the use of a diverting stoma for lower rectal carcinomas, especially in patients in poor general condition. . $^{3,4}$ 
The critics raised many questions about such recommendations. One of them is the problem with diverting stoma closure. One must understand that stoma closure is not just a simple operation reserved for young surgeons and residents but a serious procedure with quite moderate morbidity and mortality. ${ }^{5-7}$ Overall postoperative morbidity in systematic reviews is reported as high as $17 \%$, with a rate of 7.2-7.6\% for postoperative bowel obstruction, $1.4-2.0 \%$ for anastomotic leak and $1.2 \%$ for bowel perforation. ${ }^{5-8}$

Type of stoma closure depends on surgical preferences and skills. There are at least three different approaches: the anterior wall (AWT) or fold-over technique, resection with end-to-end anastomosis (RWA) or latero-lateral anastomosis, hand sewn or stapled.

The aim of our study was to evaluate morbidity and mortality after diverting stoma closure and to identify risk factors for complications of stoma closure procedure regarding various factors.

\section{Patients and methods}

We performed a retrospective cohort analysis of the patients operated in our department between 2003 and 2015. Patients with diverting ileostomy and colostomy were included. At the time of primary procedure, it was the surgeon's personal decision which type of stoma had to be made. Institutional Review Board approved the study.

Only patients after rectal cancer surgery with diverting stoma made were included in the study. Gastrografin enema or colonoscopy were performed in all patients prior to stoma closure. Patients had routine mechanical preparation of the proximal and distal bowel with the cessation of oral feeding the day before surgery. All patients underwent single-shot parenteral antibiotic treatment (cefuroxime and metronidazole) one hour prior to operation. Elementary data included age, sex, American Society of Anaesthesiologists (ASA) score at primary and stoma closure operation, and time from the primary operation to closure. Operative and postoperative data included type of operation, time until release and postoperative complications.

Two main closure techniques were used. The anterior wall technique (AWT) was performed, leaving the mesenteric side of the bowel intact and closing the bowel enterostomy in a transverse fashion by using a double-layer technique with absorbable suture material. The other technique, resection with anastomosis (RWA), represents the resection of bowel with hand-sewn entero-entero anastomosis in the same fashion.

Complications were classified according to Clavien-Dindo classification (Table 1), but there were also more specific complications accessed separately: postoperative ileus requiring reoperation, paralytic ileus that did not require reoperation, wound surgical site infection (SSI) and anastomotic leakage. ${ }^{9}$

Intestinal obstruction was defined by a combination of the following findings: abdominal distention, abdominal pain, vomiting and the presence of air-fluid levels with imaging techniques during the postoperative period. Wound infection was defined by the presence of purulent discharge, erythema, and induration of the wound. Anastomotic leakage was defined by the presence of clinical or laboratory signs of acute abdomen and was confirmed by an ultrasound, contrast enema, or computed tomography scan.

TABLE 1. Clavien-Dindo classification

\begin{tabular}{cl}
\hline Grade & Explanation \\
\hline 1 & $\begin{array}{l}\text { Any deviation from the normal postoperative course without the need for pharmacological treatment or surgical, endoscopic } \\
\text { and radiological interventions. Acceptable therapeutic regimens are: drugs as antiemetics, antipyretics, analgetics, diuretics and } \\
\text { electrolytes, and physiotherapy. This grade also includes wound infections opened at the bedside. }\end{array}$ \\
2 & $\begin{array}{l}\text { Requiring pharmacological treatment with drugs other than such allowed for grade I complications. Blood transfusions, antibiotics and } \\
\text { total parenteral nutrition are also included. }\end{array}$ \\
3 & Requiring surgical, endoscopic or radiological intervention. \\
a & Intervention under regional/local anesthesia. \\
b & Intervention under general anesthesia. \\
4 & Life-threatening complication requiring intensive care/intensive care unit management. \\
a & Single organ dysfunction. \\
b & Multi-organ dysfunction. \\
5 & Patient demise. \\
\hline
\end{tabular}


According to the time of stoma closure, we divided patients into two groups. In the first group, there were patients where we managed to close the stoma prior to eight months after rectal surgery, and in the second group, there were patients where the stoma was closed later than eight months after the initial surgery. Patients were accessed for complications during the hospitalisation, at the dismissal and at the control at the outpatient clinic 5 weeks after dismissal. None of the patients was lost.

All of the statistical analyses were performed using the SPSS 16.0 software (SPSS Inc., Chicago, Illinois, USA). Continuous data are expressed as means $\pm \mathrm{SD}$, while categorical variables are given as percentages. The Shapiro-Wilk test was used to determine whether the continuous data were normally distributed. Comparisons of continuous variables were carried out with Student's t-test for parametric data and Mann-Whitney $U$ test for nonparametric data. The Chi-square test was used for comparison of discrete variables. A p-value of $<0.05$ was considered as statistically significant.

\section{Results}

The medical records of 260 patients with stoma closure at the University Clinical Centre Maribor between January 2003 and December 2014 were identified and reviewed. We included only patients with diverting stoma after rectal cancer surgery. Basic clinical characteristics are presented in Table 2.

There was 116 colostomy and 102 ileostomy closures, 76 by AWT technique and 142 by RWA technique (Table 3). Median time to closure was 248 days (30-911 days).

TABLE 2. Clinical characteristics

\begin{tabular}{ll}
\hline $\begin{array}{l}N=218 \\
\text { Age }\end{array}$ & 64.6 years $(26-90)$ \\
Sex & \\
$\quad$ Male & $136(62 \%)$ \\
$\quad$ Female & $82(38 \%)$ \\
ASA score & \\
1 & $60(27 \%)$ \\
2 & $102(47 \%)$ \\
3 & $26(12 \%)$ \\
4 & $1(0.5 \%)$ \\
Time to closure & 248 days $(30-911)$ \\
\hline
\end{tabular}

TABLE 3. Type of stoma and closure technique

\begin{tabular}{llll} 
& Anterior Wall Sutures & Resection With Anastomosis & Together \\
\hline lleostomy & $7(7 \%)$ & $95(93 \%)$ & 102 \\
Colostomy & $69(59 \%)$ & $47(41 \%)$ & 116 \\
Together & $76(35 \%)$ & $142(65 \%)$ & 218 \\
\hline
\end{tabular}

TABLE 4. Complications according to Clavien-Dindo

\begin{tabular}{ll}
\hline Clavien- Dindo & $\mathrm{n}(\%)$ \\
0 & $164(75.2)$ \\
1 & $20(9.2)$ \\
2 & $18(8.3)$ \\
$3 \mathrm{~b}$ & $9(4.1)$ \\
$4 \mathrm{a}$ & $3(1.4)$ \\
5 & $4(1.8)$ \\
\hline
\end{tabular}

Morbidity was $24.8 \%$. Postoperative ileus (12\%) and wound infection (5\%) were the most common surgical complications. According to ClavienDindo classification, there were mostly ClavienDindo 1 and 2 (17.5\%), but there were also 16 grave complications (7.3), Clavien-Dindo $3 \mathrm{~b}$ and higher. Indications for operative treatment were postoperative ileus non-responding to conservative measurements, anastomotic leakage, and enterovaginal and enterocutaneal fistula. Mortality was $1.8 \%$. Four patients died, three after colostomy closure and one after ileostomy closure.

Table 5 demonstrates the effect of different variables on the total complication rate and separately on most severe complications Clavien-Dindo $3 \mathrm{~b}$ and higher. There was no effect on the complication rate regarding type of stoma (ileostomy vs. colostomy), closing technique (AWS vs. RWA), ASA status of the patient (ASA 1-2 vs. ASA 3-4), and patient age (less than 65 years vs. more than 65 year). The only factor influencing the complication rate was the time to stoma closure. We found that patients which had the stoma closed prior to 8 months after the formation had lower overall complication rate $(\mathrm{p}<0.05)$.

\section{Discussion}

There is no definitive consensus about the routine use of diverting stoma in rectal cancer surgery. Until today, there has been no evidence-based data that diverting stoma influences survival after rectal 
TABLE 5. The effect of different variables on complication rate

\begin{tabular}{lccc}
\hline Type of closure & AWS & RWA & p \\
\hline All complications & $16(7.3 \%)$ & $38(17.4 \%)$ & $>0.05$ \\
Severe complications & $5(2.2 \%)$ & $11(5 \%)$ & $>0.05$ \\
Age & $<65$ years & $>65$ years & $p$ \\
All complications & $23(10.5 \%)$ & $31(14.2 \%)$ & $>0.05$ \\
Severe complications & $4(1.8 \%)$ & $12(5.5 \%)$ & $>0.05$ \\
& & & \\
ASA status & ASA $1 \& 2$ & ASA 3 \& 4 & $p$ \\
All complications & $38(17.4 \%)$ & $6(3 \%)$ & $>0.05$ \\
Severe complications & $13(6 \%)$ & $3(1.3 \%)$ & $>0.05$ \\
& & & \\
Type of stoma & Ileostomy & Colostomy & $p$ \\
All complications & $27(12.4 \%)$ & $27(12.4 \%)$ & $>0.05$ \\
Severe complications & $9(4.1 \%)$ & $7(3.2 \%)$ & $>0.05$ \\
& & & $>0.05$ \\
Time to closure & $<240$ days & $>240$ days & $p$ \\
All complications & $14(6.4 \%)$ & $40(18 \%)$ & $=0.044$ \\
Severe complications & $4(1.8 \%)$ & $12(5.5 \%)$ & \\
\hline
\end{tabular}

cancer surgery, but many surgeons perform this procedure because it reduces septic consequences of a possible leak. ${ }^{10,11}$

If we presume that anastomotic leakage rate after low anterior resection for cancer is $5-10 \%$, we are creating around $90 \%$ of unnecessary stomas which need to be closed. ${ }^{12}$ One possible cause of anastomotic leakage is insufficient vascular supply. Poor vascular perfusion seems to play a key role in determining anastomotic viability. Until recently, the most common technique to evaluate tissue perfusion was the surgeon's intraoperative visual judgment based on clinical findings such as colour, pulsation and bleeding of resected margins. Unfortunately, many studies have suggested that the surgeon's clinical judgment is not enough to successfully predict the possibility of anastomotic leakage..$^{13}$ Recently, fluorescent angiography with indocyanine green has emerged as an innovative modality for intraoperative perfusion assessment. Fluorescent angiography with indocyanine green can be performed before or after intestinal resection or, alternatively, after creation of the anastomosis. ${ }^{14}$ The technique shows very promising results.

Although the closure of diverting stoma might seem as a rather simple operation, different stud- ies have shown a varying frequency and pattern of complications which could even lead to death. ${ }^{5-7}$ The problem with most of those studies is a great heterogeneity of patients, considering the indication for stoma construction, patient age, presence of comorbidities, type of stoma (ileo vs. colo) and other factors. ${ }^{15-22}$ A systematic review of 48 studies from 2009, including 6,107 patients, showed a $17.3 \%$ overall morbidity following the closure of loop ileostomy with a mortality rate of $0.4 \%$. There was considerable range in morbidity (from 3 to $38.5 \%$ ) and mortality (from 0 to $6.9 \%$ ). ${ }^{23}$ It is very interesting that such a straightforward and technically simple operation shows such different results in a relatively short time span. In our study, complication rates were rather high; morbidity was $24.8 \%$ and mortality $1.8 \%$.

We tried to identify the factors which may have contributed to relatively high complication rate and had closely investigated the patient's age, stoma type, ASA status, surgical technique of stoma closure, and time from stoma construction to closure. The data showed that neither stoma type (ileostomy or colostomy) nor surgical technique of stoma closure had impact on complication rate. The only variable we found to have an impact on postoperative morbidity is time to stoma closure.

There are still controversies about ideal time for stoma closure. There are some studies which advocate early stoma closure, but the majority of operations are still done relatively late. One must consider that the majority of rectal cancer patients are scheduled for postoperative chemotherapy which may postpone any surgical treatment. Optimal time to start chemotherapy after surgery is not well established, but it is usually accepted that it should begin within 6-8 weeks after surgery and usually for a total of about six months. It is also unknown whether adjuvant therapy in patients with rectal cancer has an impact on the morbidity of loop ileostomy closure ${ }^{24}$, but the strategy in our institution is to postpone the closure until the end of adjuvant therapy, which is approximately 8 months after initial surgery. According to our data, patients which had stoma closed prior to 8 months after primary operation had significantly less complications than patients where stoma was closed later.

Late stoma closure is connected with stoma related complications such as parastomal hernia, prolapse, retraction, peristomal dermatitis and peristomal fistula..$^{25} \mathrm{~A}$ prospective study from 2005 has shown an increase in the number of parastomal hernias, prolapses and skin irritations from the 10day follow-up to the 3-month follow-up and again 
to the 2-year follow-up. The same applied to general ostomy problems such as leakages and the need for frequent emptying. ${ }^{26} \mathrm{~A}$ prospective randomised study by Alvez et al. demonstrated a much higher number of stoma-related complications in patients whose stoma was closed after two months compared with those whose stoma was closed after only eight days. Late closure led to complications in $12 \%$ of the patients in comparison to only $1 \%$ of early closure patients. ${ }^{27}$

In a multicentre pilot study in Germany, stoma closure was performed 5.1 months after the creation on 171 patients from 17 surgical centres. ${ }^{28}$ They stated that there is no recommendation for optimal timing for stoma closure available and they started a very interesting study in 2013 where they "compare completeness of adjuvant chemotherapy after early versus late protecting stoma closure in low anterior resection for rectal cancer - CoCStom tri$\mathrm{al}^{\prime \prime} .^{28}$ They believe that early stoma closure has a beneficial effect for a patient. While we are awaiting the final results of this study, there are already some researches and articles in literature about early vs. late stoma closure.

Several prospective studies and a single randomized controlled trial have shown that closure in less than two weeks after stoma creation was associated with lower or equal morbidity compared with later closure. Thus, some authors support the early closure of temporary ileostomy performed to cover rectal anastomosis in routine clinical practice. ${ }^{25}$ On the other hand, Perez et al., performed a study on 93 patients undergoing ileostomy closure and concluded that the interval between primary operation and ileostomy closure should be no shorter than 8.5 weeks if morbidity of this procedure is to be reduced..$^{20}$

Regarding other investigated parameters, one could expect that younger patients and patients in better physical condition according to ASA status would have lower complication rate and that there could also be some differences regarding stoma type (colo- or ileostomy) and closing technique. In literature, we found many studies that did identify some connections between various other factors and complication rate after stoma closure, but the results were not uniform.

Man et al., presented their result in 2016. They recruited 213 patients with diverting ileostomy after low anterior resection. Overall complication rate was $16.4 \%$ and mortality was zero. The majority of stomas were closed after 12 weeks, mostly by stapler. They discussed and investigated possible risk factors for post closure complications: patient's age (less or more than 80 years old), influence of postoperative chemotherapy, body mass index, patient's general condition and diseases (diabetes, pre-existing respiratory or cardiac disease, operating time and anaemia. According to their data, elderly patients $(\mathrm{p}=0.002)$ and patients with a preexisting respiratory disease $(p=0.04)$ were more likely to develop postoperative complications, but elderly were defined as older than 80 years. ${ }^{29}$

In a retrospective study, Poskus et al., performed a retrospective analysis of 132 patients who underwent ileostomy closure. There were mostly patients after rectal cancer surgery but also with some other benign conditions. The complication rate was $18.2 \%$ and mortality $1.5 \%$. They found that the experience of a surgeon and preliminary condition which required diverting stoma were independent factors for complications. ${ }^{30}$

Schneider et al., published a very interesting article about surgical interventions after the ileostomy closure. In three months period, 106 patients after ileostomy closure were analysed. 12 patients required operative management due to ClavienDindo $3 b$ complications. Higher body mass index and anaemia were associated with immediate reoperations. There was no mortality. ${ }^{31}$

According to our data, ileostomy closure technique has no impact on the complication rate. Attaallah et al., investigated the postoperative course of patients treated with fold-over technique and end-to-end anastomosis as two closing techniques. They did not find any differences between the two groups of patients. ${ }^{32}$ Similar findings have been shown by Cheong et al. They concluded that fold-over technique and the conventional resection with anastomosis have similar short-term clinical outcomes for diverting ileostomy reversal. ${ }^{33}$

One of the most common and important complications of stoma closure is also surgical site infection. The reported incidence may be as high as $40 \%{ }^{34.35}$ Therefore, some authors suggest leaving the wound open, whereas others found lower infection rates after primary closure. A modification of wound closure is the so-called purse string approximation in which a circumferential approximation of the wound is performed. Providing a hole in the centre of the wound, this approximation follows the intention to drain wound liquids and therefore reduce SSI rates. ${ }^{36}$

The limitations of our study lie in its retrospective. Although the patients in a group where stoma was closed earlier had less complications, the reason for this could be that those patients had shorter or no adjuvant therapy because of less advanced 
primary disease. We also did not explore other factors that, according to some studies, might contribute to stoma closure complications like higher body mass index, anaemia and diabetes.

\section{Conclusions}

Diverting stoma closure is not a simple operation and should not be taken lightly because it is associated with significant morbidity and mortality. According to our data, the only factor that contributed to lower complication rate in our group of patients was time to stoma closure.

\section{References}

1. McArdle CS, McMillan DC, Hole DJ. Impact of anastomotic leakage on long-term survival of patients undergoing curative resection for colorectal surgery. Br J Surg 2005 92: 1150-4. doi: 10.1002/bjs.5054

2. Peeters $K C$, Tollenaar RA, Marijnen CA, Klein Kranenbarg E, Steup $W_{H}$ Wiggers $T$, et al. Risk factors for anastomotic failure after total mesorectal excision of rectal cancer. Br J Surg 2005; 92: 211-6. doi: 10.1002/bjs.4806

3. Gastinger I, Marusch F, Steinert R, Wolff S, Koeckerling F, Lippert H, Working Group 'Colon/Rectum Carcinoma'. Protective defunctioning stoma in low anterior resection for rectal carcinoma. Br J Surg 2005; 92: 1137-42. doi: 10.1002/bjs.5045

4. Matthiessen P, Hallböök O, Rutegård J, Simert G, Sjödahl R. Defunctioning stoma reduces symptomatic anastomotic leakage after low anterior resection of the rectum for cancer. A randomized multicentre trial. Ann Surg 2007; 246: 207-14. doi: 10.1097/SLA.0b013e3180603024

5. Schneider V, Lee LD, Stroux A, Buhr HJ, Ritz JP, Kreis ME, et al. Risk factors for reoperation after ileostomy reversal - results from a prospective cohort study. Int J Surg 2016; 36: 233-9. doi: 10.1016/j.ijsu.2016.10.043

6. Cipe G, Erkek B, Kuzu A, Gecim E. Morbidity and mortality after the closure of a protective loop ileostomy: analysis of possible predictors. Hepatogastroenterology 2012; 59: 2168-72. doi: 10.5754/hge12115

7. El-Hussuna A, Lauritsen M, Bülow S. Relatively high incidence of complications after loop ileostomy reversal. Dan Med J 2012; 59: A4517.

8. Sharma A, Deeb AP, Rickles AS, lannuzzi JC, Monson JR, Fleming FJ. Closure of defunctioning loop ileostomy is associated with considerable morbidity. Colorectal Dis 2013; 15: 458-62. doi: 10.1111/codi.12029

9. Clavien PA1, Barkun J, de Oliveira ML, Vauthey JN, Dindo D, Schulick RD, et al. The Clavien-Dindo classification of surgical complications: five-year experience. Ann Surg 2009; 250: 187-96. doi: 10.1097/SLA.0b013e3181b13ca2

10. Montedori A, Cirocchi R, Farinella E, Sciannameo F, Abraha I. Covering ileoor colostomy in anterior resection for rectal carcinoma. Cochrane Database Syst Rev 2010; 12; 5: CD006878. doi: 10.1002/14651858.CD006878

11. Gu WL, Wu SW. Meta-analysis of defunctioning stoma in low anterio resection with total mesorectal excision for rectal cancer: evidence based on thirteen studies. World J Surg Oncol 2015; 13: 9. doi: 10.1186/s12957014-0417-1

12. Paun BC, Cassie S, MacLean AR, Dixon E, Buie WD. Postoperative complications following surgery for rectal cancer. Ann Surg 2010; 251: 807-18. doi: 10.1097/SLA.0b013e3181dae4ed

13. Boni L, David G, Dionigi G, Rausei S, Cassinotti E, Fingerhut A. Indocyanine green-enhanced fluorescence to assess bowel perfusion during laparoscopic colorectal resection. Surg Endosc 2016; 30: 2736-42. doi: 10.1007/ s00464-015-4540-z

14. Sujatha-Bhaskar S, Jafari MD, Stamos MJ. The role of fluorescent angiography in anastomotic leaks. Surg Technol Int 2017; 25: 83-8.

15. van de Pavoordt HD, Fazio VW, Jagelman DG, Lavery IC, Weakley FL. The outcome of loop ileostomy closure in 293 cases. Int J Colorectal Dis 1987; 2: 214-7.
16. Kaiser AM, Israelit S, Klaristenfeld D, Selvindoss P, Vukasin P, Ault G, et al. Morbidity of ostomy takedown. J Gastrointest Surg 2008; 12: 437-41. doi: $10.1007 /$ s11605-007-0457-8

17. Williams LA, Sagar PM, Finan PJ, BurkeD. The outcome of loop ileostomy closure: a prospective study. Colorectal Dis 2008; 10: 460-4. doi: 10.1111/j.1463-1318.2007.01385.x

18. Mann U, Stewart PJ, Goodwin RJ, Chapuis PH, Bokey EL. Complications following closure of loop ileostomy. Aust NZ I Surg 1991; 61: 493-6. doi: 10.1111/j.1445-2197.1991.tb00275.x

19. Mansfield SD, Jensen C, Phair AS, Kelly OT, Kelly SB. Complications of loop ileostomy closure: a retrospective cohort analysis of 123 patients. World $\mathrm{J}$ Surg 2008; 32: 2101-6. doi: 10.1007/s00268-008-9669-7

20. Perez RO, Habr-Gama A, Seid VE, Proscurshim I, Sousa AH Jr, et al 2006 Loop ileostomy morbidity: timing of closure matters. Dis Colon Rectum 2008; 49: 1539-45. doi: 10.1007/s10350-006-0645-8

21. Phang PT, Hain JM, Perez-Ramirez JJ, Madoff RD, Gemlo BT. Techniques and complications of ileostomy takedown. AmJ Surg 1999; 177: 463-6. doi: 10.1016/s0002-9610(99)00091-4

22. Rathnayake MM, Kumarage SK, Wijesuriya SR, Munasinghe BN, Ariyaratne $\mathrm{MH}$, Deen KI. Complications of loop ileostomy and ileostomy closure and their implications for extended enterostomal therapy: a prospective clinical study. Int J Nurs Stud 2008; 45: 1118-21. doi: 10.1016/j.ijnurstu.2007.07.015

23. Chow A, Tilney HS, Paraskeva P, Jeyarajah S, Zacharakis E, Purkayastha S. The morbidity surrounding reversal of defunctioning ileostomies: a systematic review of 48 studies including 6,107 cases. Int J Colorectal Dis 2009; 24 711-23. doi: 10.1007/s00384-009-0660-z

24. Thalheimer A, Bueter M, Kortuem M, Thiede A, Meyer D. Morbidity of temporary loop ileostomy in patients with colorectal cancer. Dis Colon Rectum 2006; 49: 1011. doi: 10.1007/s10350-006-0541-2

25. Hindenburg T, Rosenberg J. Closing a temporary ileostomy within two weeks. Dan Med Bull 2010; 57: A4157.

26. Robertsen I, Leung E, Hughes D, Spiers M, Donnelly L, Mackenzie I, et al. Prospective analysis of stoma-related complications. Colorectal Dis 2005; 7: 279-8. doi: 10.1111/j.1463-1318.2005.00785.x

27. Alves A, Panis Y, Lelong B, Dousset B, Benoist S, Vicaut E. Randomized clinica trial of early versus delayed temporary stoma closure after proctectomy. $\mathrm{Br}$ J Surg 2008; 95: 693-8. doi: 10.1002/bjs.6212

28. Sandra-Petrescu F, Herrle F, Hinke A, Rossion I, Suelberg H, Post S, et al. CoCStom trial: study protocol for a randomised trial comparing completeness of adjuvant chemotherapy after early versus late diverting stoma closure in low anterior resection for rectal cancer. BMC Cancer 2015; 15: 923. doi: 10.1186/s12885-015-1838-0

29. Man VC, Choi HK, Law WL, Foo DC. Morbidities after closure of ileostomy: analysis of risk factors. Int J Colorectal Dis 2016; 31: 51-7. doi: 10.1007/ s00384-015-2327-2

30. Poskus E, Kildusis E, Smolskas E, Ambrazevicius M, Strupas K. Complications after Loop lleostomy Closure: A Retrospective Analysis of 132 Patients. Viszeralmedizin 2014; 30: 276-80. doi: 10.1159/000366218

31. Schneider V, Lee LD, Stroux A, Buhr HJ, Ritz JP, Kreis ME, et al. Risk factors for reoperation after ileostomy reversal - results from a prospective cohort study. Int J Surg 2016; 36: 233-9. doi: 10.1016/j.ijsu.2016.10.043

32. Attaallah W, Aktanls AO. Is the end-to-end, hand-sewn anastomosis for diverting ileostomy reversal less safe than the fold-over technique? Turk $J$ Colorectal Dis 2016; 26: 125-9. doi: 10.4274/tjcd.33602

33. Cheong J, Kang J, Kim IK, Kim NK, Sohn SK, Lee KY. Feasibility and safety of a fold-over diverting ileostomy reversal after rectal cancer surgery: casematched comparison to the resection technique. Ann Coloproctol 2014; 30: 118-21. doi: 10.3393/ac.2014.30.3.118

34. Chow A, Tilney HS, Paraskeva P, Jeyarajah S, Zacharakis E, Purkayastha S. The morbidity surrounding reversal of defunctioning ileostomies: a systematic review of 48 studies including 6,107 cases. Int J Colorectal Dis 2009; 24: 711-23. doi: 10.1007/s00384-009-0660-z

35. Milanchi, Y. Nasseri, T. Kidner, P. Fleshner S. Wound infection after ileostomy closure can be eliminated by circumferential subcuticular wound approximation. Dis Colon Rectum 2009; 52: 469-74. doi: 10.1007/ DCR.0b013e31819acc90

36. Klink CD, Wünschmann M, Binnebösel M, Alizai HP, Lambertz A, Boehm G, et al. Influence of skin closure technique on surgical site infection after loop ileostomy reversal: retrospective cohort study. Int J Surg 2013; 11: 1123-5. doi: 10.1016/j.ijsu.2013.09.003 